Mone Zaidi ORCID iD: 0000-0001-5911-9522

Daria Lizneva ORCID iD: 0000-0002-2901-4268

Kseniia Ievleva ORCID iD: 0000-0002-0177-234X

Running Head:Beyond Bone Biology

PERSPECTIVE

\title{
Beyond Bone Biology: Lessons from Team Science
}

${ }^{1}$ Mone Zaidi, ${ }^{1}$ Daria Lizneva, ${ }^{1}$ Sakshi Gera, ${ }^{1}$ Charit Taneja, ${ }^{1}$ Funda Korkmaz, ${ }^{1}$ Anisa Gumerova, ${ }^{1,2}$ Kseniia Ievleva, ${ }^{1}$ Naseer Ahmad, ${ }^{1}$ Vitaly Ryu, ${ }^{1}$ Li Sun, ${ }^{1}$ Se-Min Kim, ${ }^{1}$ Maria I. New, ${ }^{3}$ Shozeb Haider, ${ }^{1}$ Jameel Iqbal, ${ }^{4}$ Clifford Rosen and ${ }^{1}$ Tony Yuen

${ }^{1}$ The Mount Sinai Bone Program, Department of Medicine, Icahn School of Medicine at Mount Sinai, New York, NY, USA;

${ }^{2}$ Federal State Public Scientific Institution, Scientific Center for Family Health and Human Reproduction Problems, Irkutsk, Russian Federation;

${ }^{3}$ School of Pharmacy, University College London, London, UK;

${ }^{4}$ Maine Medical Center Research Institute, Scarborough, ME, USA

Correspondence: Mone Zaidi, Email: mone.zaidi@mssm.edu. Fax: 212-426-8312

Author contribution statement: M.Z. and D.L. wrote the manuscript with the input from all authors.

This article has been accepted for publication and undergone full peer review but has not been through the copyediting, typesetting, pagination and proofreading process, which may lead to differences between this version and the Version of Record. Please cite this article as doi: 10.1002/jor.24771.

This article is protected by copyright. All rights reserved. 


\begin{abstract}
Today, research in biomedicine often requires the knowledge and technologies in diverse fields. Therefore, there is an increasing need for collaborative team science that crosses traditional disciplines. Here, we discuss our own lessons from both interdisciplinary and transdisciplinary teams, which ultimately ushered us to expand our research realm beyond bone biology.
\end{abstract}

\title{
KEY WORDS
}

Bone, Osteoporosis, Biomarkers, Cell and Molecular imaging, Metabolic Bone Disease

\section{INTRODUCTION}

The cataloging of human mutations, the use of mouse genetics to recapitulate human disease, and the rapid evolution of methods in computational biology, including the ability to study the genome, transcriptome and cistrome, now at the single cell resolution, have together had a major impact on how basic biological research is translated into bedside medicine. Such has happened in bone biology with the discovery, through mouse genetics, of several medicines to treat osteoporosis and rare genetic diseases of bone. However, in achieving these strides, it has also become clear that the clinical question(s) that leads to purposeful biomedical investigation arises from the bedside. And, when the complexity of chronic diseases, such as osteoporosis, obesity and cancer, among others, has become challenging, one is prompted to resort to novel ways of examining hypotheses, studying pathophysiology, and unmasking therapeutic targets. Oftentimes to get true and meaningful answers to bedside-driven questions, one also 
needs to break through silos, cross traditional disciplines, and use unfamiliar technologies - all of this contributes to collegiality and collaboration, with the ultimate goal of ensuring rigor and integrity of data so produced.

Figure 1 shows the collaborative hierarchy of what has been popularly termed as 'team science.' It starts from uni-disciplinarity, which relates to silos of interested scientists working independently, to multidisciplinary collaborations, where groups remain independent, but interact with others, generally in sequence and in response to specific needs. However, at a higher level, interdisciplinary collaborations represent true partnerships that are interactive and collaborative, whereas the very highest echelon of team science - transdisciplinarity - represents what generally evolves into an interdependent and highly interactive team of collaborators.

While there is an evolving interest in sociology of the science behind team science $^{1-4}$, we will focus here on our own lessons from both interdisciplinary and transdisciplinary teams, which have led us to extend our remit of interest from bone biology to obesity, cancer and rare genetic diseases. We used a progressively interdisciplinary model in which we included, at various steps, expertise from basic biochemistry to structural biology to clinical oncology. Using this format, we discovered that bisphosphonates - the most commonly utilized drugs for osteoporosis and skeletal metastasis, can be repurposed to inhibit the growth of certain cancers driven by the epidermal growth factor receptor (EGFR $)^{5 ;}$. In a second longstanding effort to study the direct effects of pituitary hormones on bone $\mathrm{e}^{7-10}$, and more recently on adipose tissue and energy metabolism ${ }^{11}$, we worked as a seamless team with Cliff Rosen's group and other collaborators, to not only utilize unfamiliar technologies, but also for others to replicate

This article is protected by copyright. All rights reserved. 
and reproduce our joint data sets. Noting that new connections between bone and other tissues continue to be established ${ }^{10}$, we share our own experience to provide bone biologists with practical ways of integrating bone with other tissues towards potential therapeutic advantages.

\section{INTERDISCIPLINARY EFFORT TO RE-PURPOSE BISPHOSPHONATES FOR TREATING EGFR-DRIVEN CANCERS}

Bisphosphonates have been the mainstay of the therapy for osteoporosis, where they have been conclusively demonstrated to reduce fracture risk ${ }^{12}$. Prior to the advent to this use, bisphosphonates were used widely for the treatment of bone diseases arising from cancer, including skeletal metastasis, hypercalcemia of malignancy and osteoporosis arising from cancer therapies ${ }^{13}$. The target cell for bisphosphonate action on bone is the osteoclast, under which the drug first sequesters and is then taken up into the cell by a yet uncharacterized mechanism. The drugs mainly act to inhibit the activation of a critical enzyme, farnesyl pyrophosphate synthase (FPPS), which is responsible for geranylgeranylation of GTPases to allow enzyme and acid secretion ${ }^{14}$. By inhibiting FPPS, bisphosphonates reduce bone resorptive activity of mature osteoclasts. Commonly utilized bisphosphonates, namely alendronate, risedronate, ibandronate and zoledronic acid, which have been shown to reduce the risk of fracture at various sites, display a rank order of potency in inhibiting FPPS in vitro ${ }^{12 ; 14}$.

In addition to their prominent and well-documented skeletal actions, bisphosphonates have been shown to directly kill certain cancer cells and improve survival in people. The most compelling data sets arise in patients with breast and colon 
cancers. Bisphosphonates utilized in early breast cancer without evidence of skeletal metastasis have been shown to reduce isolated tumor cell burden in bone marrow ${ }^{15-17}$. Furthermore, in patient subgroups in the ABSCG-12, ZO-FAST and AZURE breast cancer trials, zoledronic acid was found to significantly improved recurrence- and disease-free survival $^{18-20}$. Furthermore, several epidemiologic studies have documented a reduced incidence of colon and breast cancer in women who are on bisphosphonate therapy for osteoporosis ${ }^{21-23}$, and at least one study has shown a survival benefit in patients who remain on drug following their cancer diagnosis ${ }^{21}$.

To us bone biologists, these sets of compelling studies with a bone-active drug posed the question whether bisphosphonates had additional actions on yet uncharacterized molecular targets, which could be the basis of their potential repurposing as anti-cancer agents. We started with a team of two (M.Z. and L.S.), and recruited a colleague knowledgeable in genomics (J.I.), who helped us with an unbiased search for other potential targets using the recently developed connectivity map (C$\mathrm{MAP}^{24}$. This in silico tool, which connects genes, diseases and drugs, has now been extensively utilized to re-purpose 'old' drugs for new uses. Namely, the anticonvulsant topiramate is now being tested for inflammatory bowel disease ${ }^{25}$, and trifuloperazine-like agents are being evaluated for the therapy of therapy-resistant, EGFR-driven non-smallcell lung cancers (NSCLC) ${ }^{26}$. We are also beginning, based on C-MAPping a gene signature from a mouse model of Gaucher disease, to evaluate two anti-schistosomal agents, which may bypass the effects of glucocerebrosidase deficiency in Gaucher disease $^{27}$.

This article is protected by copyright. All rights reserved. 
With the inclusion of expertise in computation, we developed a bisphosphonate gene signature consisting of the most tightly associated genes with bisphosphonate action on the osteoclast, and interrogated C-MAP with this signature ${ }^{5}$. The top two hits were anti-cancer agents, 1,5-isoquinolinediol (a PARP inhibitor) and a first-generation EGFR inhibitor, AG-1478. In parallel, pathway analysis using KEGG highlighted associated pathways downstream of EGFR activation ${ }^{5 ;}{ }^{6}$. This data set led to the next question whether there is a relationship between bisphosphonates and cancers driven by EGFRs. This required expertise in oncology, and we therefore entered into collaboration with a cancer biologist (Dr. Goutham Narla, University of Michigan) and clinical oncologist (Dr. Matthew Galsky, Mount Sinai), to begin exploring potential uses for bisphosphonates in lung and breast cancers.

Lung cancer causes nearly a third of all cancer-related deaths worldwide estimated to be $\sim 160,000$ annually. A third of all NSCLCs are driven by activating mutations in the EGFR, prominently an L858R point mutation and a deletion mutation in exon 19 ( $\Delta 746-750)$. These mutations do not only cause unrestricted oncogenic signaling by allowing the $\mathrm{C} \alpha$ helix to collapse into the kinase domain, but also render the cancers sensitive to the two most commonly utilized first-line tyrosine kinase inhibitors, gefitinib and erlotinib ${ }^{28}$. However, with either agent, there is recurrence due to resistant mechanisms ${ }^{29}$, about half of which have been attributed to a second-site mutation in the kinase domain, notably $\mathrm{T} 90 \mathrm{M}^{30}$.

With the assistance of our cancer collaborators, we could show that nitrogencontaining bisphosphonates (alendronate, ibandronate, risedronate, icandronate and zoledronic acid) caused apoptosis and cell cycle arrest in human NSCLC cell lines

This article is protected by copyright. All rights reserved. 
bearing the respective EGFR mutations, namely H3255 (with the L858R mutation) and HCC827 (with the $\Delta 746-750$ deletion) ${ }^{5 ;}$. In contrast, the 'older' bisphosphonates that did not have an amino group (etidronate, clodronate, tiludronate and pamidronate) failed to kill cells - suggesting a structure-activity relationship, rather than a mass effect. Specifically, two agents zoledronic acid (used mainly in the US and Europe) and minodronic acid (used mainly in Asia), which had similar ring structures and two nitrogen atoms, displayed almost identical actions in reducing cell viability.

Exploration of the precise molecular mechanisms of action of bisphosphonates on EGFRs meant that we add a structural biologist to our team (S.H.). We hypothesized that, as bisphosphonates shared two phosphate groups with ATP, the molecules will likely bind with a weak affinity to the kinase domain of the EGFR ${ }^{5 ; 6}$. We performed computational modeling, including molecular dynamics and anisotropic network modeling, to establish that bisphosphonates could dock stably into the kinase domain of the EGFR. Using the crystal structure of the EGFR kinase domain, we found that zoledronic acid forms a bond via water with residue T790 of the EGFR and its imidazole ring docks into the adenine binding site. Through our collaboration with a biochemist (T.Y.), we developed a protein thermal shift assay to conclusively establish binding of bisphosphonates $^{6}$. There was a clear shift in the melting temperature of recombinant EGFR in the presence of either bisphosphonate. Importantly, this thermal shift was abolished when the putative bisphosphonate-binding sites of EGFR were mutated by site-directed mutagenesis ${ }^{5 ;}$. We also explored signaling pathways downstream of the kinase pocket, and found that tyrosine phosphorylation of all downstream residues, together with associated signaling cascades, namely Stat3/5, Akt, NFkB and Erk1/2 were

This article is protected by copyright. All rights reserved. 
attenuated substantially ${ }^{5 ;}$. A global reduction in EGFR signaling was consistent with the action of bisphosphonates at the kinase domain.

We studied whether bisphosphonates could synergize with erlotinib or gefitinib to mount a more profound effect on tumor cell viability. Through our continued structural biology collaboration, we were able to document, by computational modeling, that the two drugs together bound to the EGFR kinase domain, but with different binding modes than when each drug was docked on its own ${ }^{5}$. The concept that one 'big drug' could work better than two small drugs led to the testing of synergy both in colony-forming assays and xenotransplant studies. We found that bisphosphonates used together with erlotinib caused greater reductions in colony formation than each drug alone ${ }^{5}$. We also showed that while each drug inhibited tumor growth and caused apoptosis, the two drugs together resulted in profound tumor regression ${ }^{5}$. Of note is that EGFR-negative colon cancer cells, when similarly tested did not respond to bisphosphonate action, and that inhibiting EGFR expression in H3255 and HCC827 cells significantly attenuated bisphosphonate-induced cell killing, whereas inhibiting FPPS did not ${ }^{5 ; 6}$ - this confirmed a primary action of bisphosphonates on cell viability via the EGFR.

We also explored whether bisphosphonates could be used in patients with the second site mutation, T790M. This mutation creates a steric clash with a nearby M766 residue in the L858R-T790M double mutant resulting in a $20^{\circ}$ rigid rotation of the $\mathrm{C} \alpha$ helix so that erlotinib and gefitinib are unable to bind ${ }^{5 ; 6}$. However, the interaction with bisphosphonates is preserved, testified by the robust inhibition of cell viability in a cell line that harbored both the driver (L858R) and resistance (T790M) mutations.

This article is protected by copyright. All rights reserved. 
These findings together suggest that the widely-utilized bone-active bisphosphonates could potentially be repurposed as anti-cancer agents for tumors that are driven primarily by either overexpression or constitutive activation of the EGFR family of receptors, such as NSCLC, breast, colon, gastric, and head and neck cancers. From our combination therapy dataset, it seems possible that bisphosphonates could become valuable adjunctive therapies for patients on tyrosine kinase inhibitors, particularly in cases of resistant disease, where patients follow a downhill course. Furthermore, our prevention model documents robust effects of bisphosphonates on HER2-driven breast cancer ${ }^{5}$. And finally, having teamed up with a medicinal chemist (Dr. Michael Ohlmeyer, Mount Sinai), we are designing a new class of drugs, which we predict are as, if not more, potent that the current tyrosine kinase inhibitors, and may also overcome the early resistance. Our 'farm approach' for interdisciplinary collaboration developed as key questions arose (Figure 2) appeared therefore to deliver a strong platform for repurposing a common class of drugs for important therapeutic uses.

\section{TRANSDISCIPLINARY EFFORTS LEAD TO THE DISCOVERY OF FSH ACTION ON BONE AND FAT}

In 2003, we provided the first evidence that pituitary hormones, otherwise thought to have unitary associations with target tissues, could act directly on the skeleton through G-protein-coupled receptors ${ }^{7}$. Notably, shortly after discovery of neural circuits for the regulation of bone mass through the sympathetic nervous system ${ }^{31}$, we found that both thyroid-stimulating hormone (TSH) and follicle-stimulating hormone (FSH), thought solely to regulate thyroxine secretion from the thyroid gland and ovarian estrogen production, respectively, could act on osteoclasts to regulate bone resorption ${ }^{7 ; 8}$. Since 
then not only has every pituitary hormone, namely FSH, TSH, growth hormone, adenocorticotrophic hormone (ACTH) and prolactin, as well as posterior pituitary neuropeptides, oxytocin and vasopressin, been shown to act directly on bone, but also that certain of these hormones are produced by bone cells to exert potential autocrine and paracrine actions $^{7-10 ; 32-39}$. Subsequently, clinical associations between pituitary hormones and bone, body composition, and energy metabolism have been documented using various cohorts across the globe [reviews: Refs 9; 40].

Collectively, these studies have not only yielded new insights into integrative physiology specifying a pituitary-bone axis and pituitary-metabolic circuits of medical significance, but have also led to efforts at remapping single-hormone hypotheses for the pathophysiology of human osteoporosis. Thus, it is becoming increasingly accepted that the osteoporosis of hyperthyroidism and hypogonadism, which was solely attributed to changes in thyroxine and estrogen levels, also have contributions from reduced TSH and elevated FSH levels respectively ${ }^{9 ;}$. Likewise, the osteoporosis of pregnancy and lactation and that resulting from chronic hypernatremia are being attributed to increased levels of oxytocin and vasopressin ${ }^{9 ;} 40$. Here, we will focus on the role of FSH in causing bone loss and adiposity - studies that were performed and validated in a truly transdisciplinary manner and have formed the framework for the development of an antiFSH antibody as a potential therapeutic for both osteoporosis and obesity.

Indeed, it is clear from the Study of Women's Health Across the Nation (SWAN) that women entering menopause display an accelerated phase of bone loss, with $50 \%$ of bone loss occurring within the first 5 years of menopause. This is accompanied by the onset of visceral obesity, disrupted energy homeostasis, and reduced physical activity in

This article is protected by copyright. All rights reserved. 
millions of women worldwide ${ }^{41-48}$. The bone loss has been attributed solely to estrogen deficiency since the time Fuller Albright described post-menopausal osteoporosis ${ }^{49}$. However, a more careful look at the SWAN data indicates that the most rapid rates of bone loss occur $\sim 3$ years prior to the onset of the last menstrual period ${ }^{47 ;} 48$. During this period in a woman's life (between 42 and 54 years), irrespective of ethnicity, estrogen levels are relatively normal while FSH levels are rising ${ }^{41}$. We therefore surmised that the rapid rates of bone loss cannot be attributed to hypoestrogenemia and that elevated FSH levels may play a causal role.

We found FSH receptors (FSHRs) on osteoclasts from various species, including human CD14-derived cells, which were coupled to a $\mathrm{G} \alpha_{\mathrm{i} 2}$ protein (instead of coupling to a $G \alpha_{s}$ protein in ovarian follicular cells) ${ }^{8 ;} 50 ; 51$. Activation of the FSHR resulted in increased formation, activity and survival of osteoclast by synergizing the MAP kinase, Akt and NFkB pathways ${ }^{8}$. Furthermore, we separated the potentially confounding effects of estrogen from the pro-resorptive actions of FSH using mice lacking FSH $\beta^{8}$. Specifically, mice haploinsufficient in FSH $\beta$, namely $\mathrm{Fshb}^{+/-}$mice, showed no evidence of ovarian dysfunction, whereas there was a significant increase in bone mass due to suppressed osteoclastic bone removal ${ }^{8}$. Collectively, the data documented a direct action of FSH to increase the resorption of bone, leading to the hypothesis that some of the bone loss seen in the late perimenopause could arise from elevated serum FSH in the face of normal estrogen levels ${ }^{41 ;} 46$. The question was whether blocking the action of FSH during this period would prevent postmenopausal bone loss.

These initial observations led us to our structural biology collaborator, who used the crystal structure of the human FSHR-FSH $\beta$ complex to model and fine map the This article is protected by copyright. All rights reserved. 
equivalent mouse complex ${ }^{52 ;}$. This led us to use a 13-amino-acid-long sequence of FSH $\beta$ as the target for our initial antibody. Our approach for using a highly-targeted antibody to the ligand had several advantages. First, one could titrate antibody levels so as to spare the ovaries by leaving enough circulating FSH unblocked. Second, the human and mouse peptide sequences differed in just two amino acids, so that even a humanized antibody would likely recognize mouse FSH, thus enabling its future testing in mouse models. Third, blocking the FSHR-binding domain of FSH $\beta$ using a large immunoglobulin molecule is likely to prevent FSH access into the small binding pocket of the FSHR ${ }^{52}$. Thus, our polyclonal antibody reduced the fall of bone density that was triggered upon ovariectomy in mice ${ }^{53 ;}$, providing proof-of-concept for a potential therapeutic use in postmenopausal osteoporosis. We also noted that the antibody not only inhibited bone resorption in these ovariectomized mice, but surprisingly also stimulated bone formation ${ }^{52 ;}{ }^{33}$. Noting that we did not find FSHRs on mature osteoblasts, we documented expression of these receptors on mesenchymal stem cells $s^{53}$, which are also precursors for adipocytes. We therefore questioned, on the basis of preliminary data that, if an anti-FSH antibody was pro-osteoblastic, could it also possess anti-adipocytic actions?

The anti-FSH antibody did indeed reduce body fat on dual energy X-ray absorptiometry (DXA), in addition to increasing bone mass ${ }^{11}$. This dataset became the beginning of a 5-year-long, highly productive and mutually-rewarding transdisciplinary collaboration with Dr. Clifford Rosen - we jointly asked the question whether our antiFSH antibody could build bone and reduce body fat. In doing so, key data sets were replicated in Dr. Rosen's lab using either the same or different technologies ${ }^{11 ; 55}$. We each 
found that following ovariectomy or after being fed with a high-fat diet, mice receiving the anti-FSH antibody lost body fat, and that this was independent of food intake that was somewhat increased ${ }^{11}$. Both our groups used DXA, quantitative NMR and direct weight measurements to document these effects in both male and female mice of different ages ${ }^{11}$. The need for further confirmation, particularly as both our groups were beginning to tread into a new field of obesity and metabolism, was to have the fundamental premise validated, and we did so by including Dr. X. Edward Guo (Columbia University), who replicated our data set using micro-CT imaging. He found a dramatic reduction in fat mass in the visceral and subcutaneous compartments of antibody-treated mice, and further, that this effect phenocopied the effect of Fshr haploinsufficiency ${ }^{11}$. Overall, this triple validation strategy of key data through an extended collaboration provided the most robust evidence for an anti-adiposity action of our FSH antibody. Please refer to of our Nature paper, which attributes each experiment to a given principal investigator in order to ensure transparency ${ }^{11}$.

We also established specificity of FSH blockade through a collaboration with Dr. Henrik Molina at the Rockefeller University Proteomics Resource Center. Mass spectrometry documented FSH in eluates from anti-FSH-antibody-immobilized columns ${ }^{11}$. Blocking action was confirmed using ThermoCells that report the activation of uncoupling protein 1 (UCP1), a mitochondrial protein involved in thermogenesis. Pharmacokinetic studies in mice documenting high enough antibody concentration in the serum provided further validation. The presence of FSHRs on adipocytes, albeit at an 8fold lower level than the ovaries, was confirmed complementarily by quantitative PCR, Sanger sequencing of the full length receptors from primary adipocytes and 3T3.L1 cells, 
and functional assays on regulation of downstream gene program ${ }^{11}$. We also collaborated with Dr. Aaron Hsueh (Stanford University), as part of our transdisciplinary approached. His group established, using a fluorescently-labeled $\mathrm{FSH}$ molecule $\mathrm{FSH}-\mathrm{CH}$, in vivo FSH binding to adipose tissue that was displaceable by unlabeled $\mathrm{FSH}^{52}$. We further found that FSH inhibited the $\beta 3$ adrenergic signaling pathway, and that the FSHR was coupled (as in bone) to a pertussis-toxin-sensitive $\mathrm{G} \alpha_{\mathrm{i}}{ }^{11}$. Xenotransplant studies, wherein ThermoCells were injected into nude mice, revealed marked increases in UCP1 expression in vivo, thus suggesting that the FSH pathway was anti-thermogenic ${ }^{11}$. Blocking FSH with our antibody thus induced thermogenic adipose tissue in vivo ${ }^{11}$.

To be scientifically meaningful, studies needed not only to be repeated and reproduced in other labs (that we heavily relied upon), but also to be replicated using other technologies. To study the induction of thermogenic adipose tissue, we utilized complementary technologies at various labs. At Mount Sinai, we injected ThermoMice, which express Luc2/tdTomato driven by the Ucp1 promoter, with antibody and used the IVIS platform to assess luminescence in vivo - strong signals were seen in areas with predominantly brown or white adipose tissue ${ }^{11}$. This suggested not only brown fat activation, but also the conversion of white adipose tissue to energy-producing beige adipose tissue. Experiments with ThermoMice were replicated in Cliff Rosen's lab under thermo-neutral conditions. Second, white-to-beige transition (or 'beiging') induced by our anti-FSH antibody given to mice on a high-fat diet was further explored using traditional immunocytochemistry for UCP1, as well as by quantitative PCR for a host of brown fat genes, such as Ucp1, Cidea, Cox7a and Cox8, that were all elevated in white fat $^{11}$. Third, as beiging is associated with increased mitochondrial bioenergetics, we used 
the $\mathrm{PhAM}$ mouse, which reports mitochondria. We documented marked increases in mitochondrial density in all fat depots ${ }^{11}$. Furthermore, to study metabolic effects, Cliff Rosen performed indirect calorimetry on mice on a high-fat diet that were treated with anti-FSH antibody. This study confirmed increases in energy expenditure and oxygen utilization, but with evidence for increased physical activity ${ }^{11}$. Finally, to determine whether the enhanced physical activity noted with our antibody contributed to increased energy expenditure, we collaborated with Dr. Jan van Klinken (University of Leiden), a biomathematician and an expert in analyzing metabolic cage data. The raw dataset from metabolic cages was analyzed rigorously using penalized spline regression, which allows the separation of basal and physical-activity-induced increases in metabolic rate. We found, through this important collaboration, that the increased energy expenditure induced with the anti-FSH antibody was occurring independently of physical activity, and likely arose from beiging and brown adipose tissue activation.

In summary, we have found through an extensively validated set of data that FSH inhibition stimulates bone formation, inhibits bone resorption, reduces body fat and converts white adipose tissue to thermogenic 'beige' adipose tissue. Our transdisciplinary collaborative program, besides providing valuable expertise in areas beyond our own breadth, also represents our investigative team's profound effort towards ensuring rigor. It provides an example of exceptional level of transparency - free exchange of reagents, raw data sets, Excel spreadsheets, and cross-confirmation at all levels. Candid discussions about reproducibility and replicability led to consensus data being submitted for publication. We term this "contemporaneous replication" - a level of rigor that, we believe, contributed to our recent NIH U19 funding ${ }^{11 ;} 55$.

This article is protected by copyright. All rights reserved. 
As noted above, there is clear evidence that osteoporosis and obesity track together at menopause. Furthermore, there is an evolving consensus that obesity itself may predispose to bone loss. However, the potential clinical application of our findings is based on recent compelling human evidence from a urology group in Denmark. Østergren et al. asked the question whether FSH contributes to human obesity. In an interventional clinical trial, wherein 58 treatment-naïve prostate cancer patients underwent subcapsular orchiectomy or were given a GnRH analog triptorelin, in both instances to reduce testosterone levels to near-zero ${ }^{56}$. Notably, orchiectomized patients with high FSH and LH levels had a higher body weight, total fat mass, and subcutaneous adipose tissue, with a trend towards higher visceral adipose tissue ${ }^{56}$. This study lays down the firm basis for the potential for an anti-FSH therapy for obese people. Towards this, we have developed monoclonal antibodies to the human and mouse FSH $\beta$ sequences. These antibodies have now been humanized and await affinity maturation before entering the commercialization pipeline.

\section{OUR LESSONS}

Being involved for the past decade in what is now popularly termed 'team science'- both interdisciplinary and transdisciplinary - we find that the whole has always been greater than the sum of the parts. We initially laid out the framework of our focus on groundbreaking research that served as the fundamental catalyst in the development of our collaborations. We discovered that in establishing a multidisciplinary scientific team, where individuals share collective identity, are interdependent and mutually committed to the project, and take shared accountability for the outcome, the most important attribute was team "synergy". This synergy allowed each entity to come together and glean the 
necessary intellectual capital needed - building on and expanding strengths, while simultaneously overcoming or filling gaps and perceived weaknesses. Team development requires personal and collective maturity and internal acceptance of the fact that, in order to achieve scientific breakthroughs, we need to operate above and beyond our personal egos and ambitions. It takes time, trust, mutual respect, and a collective vision.

In building our team, we faced and addressed challenges. The average timeframe to complete an NIH-funded project is 3-5 years. However, the formation of a mature team can take a few years. Therefore, a team may sometimes operate at full capacity only $2 / 3$ of the allocated time. The individualistic culture in academic medicine focuses on the perceived "independence" of individual investigators, and seems to perpetuate the fear that more than one member of the team will be credited for their work. Multicultural and transgenerational scientific environments are associated with complicated communication styles due to differing backgrounds and a tendency to stereotype. While moving from self-identity to group identity and from independence to interdependence, ongoing issues of status, power and autonomy can be distractors and must be managed thoughtfully. Therefore, while some would state "I make a pretty good team," others might say that "sometimes I think that the collaborative process would work better without you."

There is no single universal solution as to how to overcome these barriers; however, we found that it was crucially important to keep the team focused on their role in the "big picture". We found that by maintaining transparency, delineating and continuously reaffirming roles and responsibilities by being open through frequent communication, we could be successful. These tenets have helped build trust and lasting 
relationships with our collaborators, with an added benefit of ensuring rigor and integrity of our datasets.

Notably, for us the gain of new knowledge and evolution of its therapeutic implications have been profound. Biologically, it has allowed out-of-the-box concepts to emerge and mature - and this has led to novel testable hypotheses, such as the concept of pituitary-metabolic circuitry. It has also allowed, and importantly encouraged, diversification of our interests - from bone biology to obesity, metabolism, and cancer. Moreover, our 'team science' resulted in the submission and subsequent award of a U19 application, supported by National Institute of Aging (NIA). This opportunity has allowed us for the smooth transition needed to foster scientific independence of three of our younger principal investigators - namely, T.Y., J.I. and D.L.

As we are facing the emerging changes in contemporary science, including globalization and greater advances in technology, it is crucial for NIH and academic institutions to provide more support for the integrative 'team science' approach. It is our hope that interdisciplinary and transdisciplinary collaborations will continue to expand the scope of bone biology research to a much more provocative organismal front.

\section{ACKNOWLEDGEMENTS}

M.Z., T.Y., J.I. and D.L. are grateful to the National Institutes of Health for grant support, namely R01 AG40132 (M.Z.), R01 AR67066 (M.Z.), R01 DK113627 (M.Z. and J.I.), and U19 AG60917 (M.Z., J.I., T.Y., and D.L.). M.I.N. is supported by the Maria I. New Children’s Hormone Research Foundation.

This article is protected by copyright. All rights reserved. 


\section{REFERENCES}

1. Hall KL, Feng AX, Moser RP, et al. 2008. Moving the science of team science forward: collaboration and creativity. Am J Prev Med 35:S243249.

2. Masse LC, Moser RP, Stokols D, et al. 2008. Measuring collaboration and transdisciplinary integration in team science. Am J Prev Med 35:S151160.

3. Stokols D, Hall KL, Taylor BK, et al. 2008. The science of team science: overview of the field and introduction to the supplement. Am J Prev Med 35:S77-89.

4. Stokols D, Misra S, Moser RP, et al. 2008. The ecology of team science: understanding contextual influences on transdisciplinary collaboration. Am J Prev Med 35:S96-115.

5. Stachnik A, Yuen T, Iqbal J, et al. 2014. Repurposing of bisphosphonates for the prevention and therapy of nonsmall cell lung and breast cancer. Proceedings of the National Academy of Sciences of the United States of America 111:17995-18000.

6. Yuen T, Stachnik A, Iqbal J, et al. 2014. Bisphosphonates inactivate human EGFRs to exert antitumor actions. Proceedings of the National Academy of Sciences of the United States of America 111:17989-17994. 
7. Abe E, Marians RC, Yu WQ, et al. 2003. TSH is a negative regulator of skeletal remodeling. Cell 115:151-162.

8. Sun L, Peng Y, Sharrow AC, et al. 2006. FSH directly regulates bone mass. Cell 125:247-260.

9. Zaidi M. 2007. Skeletal remodeling in health and disease. Nat Med 13:791-801.

10. Zaidi M, Yuen T, Sun L, et al. 2018. Regulation of Skeletal Homeostasis. Endocr Rev 39:701-718.

11. Liu P, Ji $\mathrm{Y}$, Yuen $\mathrm{T}$, et al. 2017. Blocking FSH induces thermogenic adipose tissue and reduces body fat. Nature 546:107-112.

12. Pazianas M, Epstein S, Zaidi M. 2009. Evaluating the antifracture efficacy of bisphosphonates. Rev Recent Clin Trials 4:122-130.

13. Russell RG. 2011. Bisphosphonates: the first 40 years. Bone 49:2-19.

14. Dunford JE, Thompson K, Coxon FP, et al. 2001. Structure-activity relationships for inhibition of farnesyl diphosphate synthase in vitro and inhibition of bone resorption in vivo by nitrogen-containing bisphosphonates. J Pharmacol Exp Ther 296:235-242.

15. Gnant M, Mlineritsch B, Schippinger W, et al. 2009. Endocrine therapy plus zoledronic acid in premenopausal breast cancer. $\mathrm{N}$ Engl $\mathrm{J}$ Med 360:679-691.

This article is protected by copyright. All rights reserved. 
16. Aft R, Naughton M, Trinkaus K, et al. 2010. Effect of zoledronic acid on disseminated tumour cells in women with locally advanced breast cancer: an open label, randomised, phase 2 trial. Lancet Oncol 11:421-428.

17. Rack B, Juckstock J, Genss EM, et al. 2010. Effect of zoledronate on persisting isolated tumour cells in patients with early breast cancer. Anticancer Res 30:1807-1813.

18. Coleman R, Gnant M, Morgan G, et al. 2012. Effects of bone-targeted agents on cancer progression and mortality. J Natl Cancer Inst 104:10591067.

19. Coleman RE, Marshall H, Cameron D, et al. 2011. Breast-cancer adjuvant therapy with zoledronic acid. N Engl J Med 365:1396-1405.

20. Gnant M, Mlineritsch B, Stoeger H, et al. 2011. Adjuvant endocrine therapy plus zoledronic acid in premenopausal women with early-stage breast cancer: 62-month follow-up from the ABCSG-12 randomised trial. Lancet Oncol 12:631-641.

21. Pazianas M, Abrahamsen B, Eiken PA, et al. 2012. Reduced colon cancer incidence and mortality in postmenopausal women treated with an oral bisphosphonate--Danish National Register Based Cohort Study. Osteoporos Int 23:2693-2701.

22. Rennert G, Pinchev M, Rennert HS, et al. 2011. Use of bisphosphonates and reduced risk of colorectal cancer. J Clin Oncol 29:1146-1150. 
23. Sendur MA, Aksoy S, Yaman S, et al. 2012. Demographic and clinicopathological characteristics of breast cancer patients with history of oral alendronate use. Med Oncol 29:2601-2605.

24. Lamb J, Crawford ED, Peck D, et al. 2006. The Connectivity Map: using gene-expression signatures to connect small molecules, genes, and disease. Science 313:1929-1935.

25. Dudley JT, Sirota M, Shenoy M, et al. 2011. Computational repositioning of the anticonvulsant topiramate for inflammatory bowel disease. Sci Transl Med 3:96ra76.

26. Sangodkar J, Dhawan NS, Melville H, et al. 2012. Targeting the FOXO1/KLF6 axis regulates EGFR signaling and treatment response. $\mathrm{J}$ Clin Invest 122:2637-2651.

27. Yuen T, Iqbal J, Zhu LL, et al. 2012. Disease-drug pairs revealed by computational genomic connectivity mapping on GBA1 deficient, Gaucher disease mice. Biochemical and Biophysical Research Communications 422:573-577.

28. Herbst RS, Heymach JV, Lippman SM. 2008. Lung cancer. N Engl J Med 359:1367-1380.

29. Nishino M, Jackman DM, Hatabu $\mathrm{H}$, et al. 2011. Imaging of lung cancer in the era of molecular medicine. Acad Radiol 18:424-436.

This article is protected by copyright. All rights reserved. 
30. Rotow J, Bivona TG. 2017. Understanding and targeting resistance mechanisms in NSCLC. Nat Rev Cancer 17:637-658.

31. Takeda S, Elefteriou F, Levasseur R, et al. 2002. Leptin regulates bone formation via the sympathetic nervous system. Cell 111:305-317.

32. Colaianni G, Di Benedetto A, Zhu LL, et al. 2011. Regulated production of the pituitary hormone oxytocin from murine and human osteoblasts. Biochemical and Biophysical Research Communications 411:512-515.

33. Colaianni G, Sun L, Di Benedetto A, et al. 2012. Bone marrow oxytocin mediates the anabolic action of estrogen on the skeleton. J Biol Chem 287:29159-29167.

34. Di Benedetto A, Sun L, Zambonin CG, et al. 2014. Osteoblast regulation via ligand-activated nuclear trafficking of the oxytocin receptor. Proceedings of the National Academy of Sciences of the United States of America 111:16502-16507.

35. Tamma R, Colaianni G, Zhu LL, et al. 2009. Oxytocin is an anabolic bone hormone. Proceedings of the National Academy of Sciences of the United States of America 106:7149-7154.

36. Tamma R, Sun L, Cuscito C, et al. 2013. Regulation of bone remodeling by vasopressin explains the bone loss in hyponatremia. Proceedings of the National Academy of Sciences of the United States of America 110:18644-18649.

This article is protected by copyright. All rights reserved. 
37. Zaidi M, Sun L, Robinson LJ, et al. 2010. ACTH protects against glucocorticoid-induced osteonecrosis of bone. Proceedings of the National Academy of Sciences of the United States of America 107:8782-8787.

38. Baliram R, Chow A, Huber AK, et al. 2013. Thyroid and bone: macrophage-derived TSH-beta splice variant increases murine osteoblastogenesis. Endocrinology 154:4919-4926.

39. Baliram R, Latif R, Morshed SA, et al. 2016. T3 Regulates a Human Macrophage-Derived TSH-beta Splice Variant: Implications for Human Bone Biology. Endocrinology 157:3658-3667.

40. Zaidi M, New MI, Blair HC, et al. 2018. Actions of pituitary hormones beyond traditional targets. J Endocrinol 237:R83-R98.

41. Randolph JF, Jr., Sowers M, Gold EB, et al. 2003. Reproductive hormones in the early menopausal transition: relationship to ethnicity, body size, and menopausal status. J Clin Endocrinol Metab 88:1516-1522.

42. Recker R, Lappe J, Davies K, et al. 2000. Characterization of perimenopausal bone loss: a prospective study. J Bone Miner Res 15:1965-1973.

43. Recker R, Lappe J, Davies KM, et al. 2004. Bone remodeling increases substantially in the years after menopause and remains increased in older osteoporosis patients. J Bone Miner Res 19:1628-1633.

This article is protected by copyright. All rights reserved. 
44. Sowers M, Eyre D, Hollis BW, et al. 1995. Biochemical markers of bone turnover in lactating and nonlactating postpartum women. J Clin Endocrinol Metab 80:2210-2216.

45. Sowers $\mathrm{M}$, Zheng $\mathrm{H}$, Tomey $\mathrm{K}$, et al. 2007. Changes in body composition in women over six years at midlife: ovarian and chronological aging. J Clin Endocrinol Metab 92:895-901.

46. Sowers MR, Finkelstein JS, Ettinger B, et al. 2003. The association of endogenous hormone concentrations and bone mineral density measures in pre- and perimenopausal women of four ethnic groups: SWAN. Osteoporos Int 14:44-52.

47. Sowers MR, Greendale GA, Bondarenko I, et al. 2003. Endogenous hormones and bone turnover markers in pre- and perimenopausal women: SWAN. Osteoporos Int 14:191-197.

48. Sowers MR, Jannausch M, McConnell D, et al. 2006. Hormone predictors of bone mineral density changes during the menopausal transition. J Clin Endocrinol Metab 91:1261-1267.

49. Albright F. 1947. Osteoporosis. Ann Intern Med 27:861-882.

50. Robinson LJ, Tourkova I, Wang Y, et al. 2010. FSH-receptor isoforms and FSH-dependent gene transcription in human monocytes and osteoclasts. Biochemical and Biophysical Research Communications 394:12-17. 
51. Sun L, Zhang Z, Zhu LL, et al. 2010. Further evidence for direct proresorptive actions of $\mathrm{FSH}$. Biochemical and Biophysical Research Communications 394:6-11.

52. Ji Y, Liu P, Yuen T, et al. 2018. Epitope-specific monoclonal antibodies to FSHbeta increase bone mass. Proceedings of the National Academy of Sciences of the United States of America 115:2192-2197.

53. Zhu LL, Blair H, Cao J, et al. 2012. Blocking antibody to the beta-subunit of FSH prevents bone loss by inhibiting bone resorption and stimulating bone synthesis. Proceedings of the National Academy of Sciences of the United States of America 109:14574-14579.

54. Zhu LL, Tourkova I, Yuen T, et al. 2012. Blocking FSH action attenuates osteoclastogenesis. Biochemical and Biophysical Research Communications 422:54-58.

55. Rosen CJ, Zaidi M. 2017. Contemporaneous reproduction of preclinical science: a case study of FSH and fat. Ann N Y Acad Sci 1404:17-19.

56. Ostergren PB, Kistorp C, Fode M, et al. 2017. Luteinizing HormoneReleasing Hormone Agonists are Superior to Subcapsular Orchiectomy in Lowering Testosterone Levels of Men with Prostate Cancer: Results from a Randomized Clinical Trial. J Urol 197:1441-1447.

\section{LEGENDS TO FIGURES}

This article is protected by copyright. All rights reserved. 
Figure 1: Collaborative Hierarchy of Team Science.

\section{COLLABORATIVE HIERARCHY IN TEAM SCIENCE}

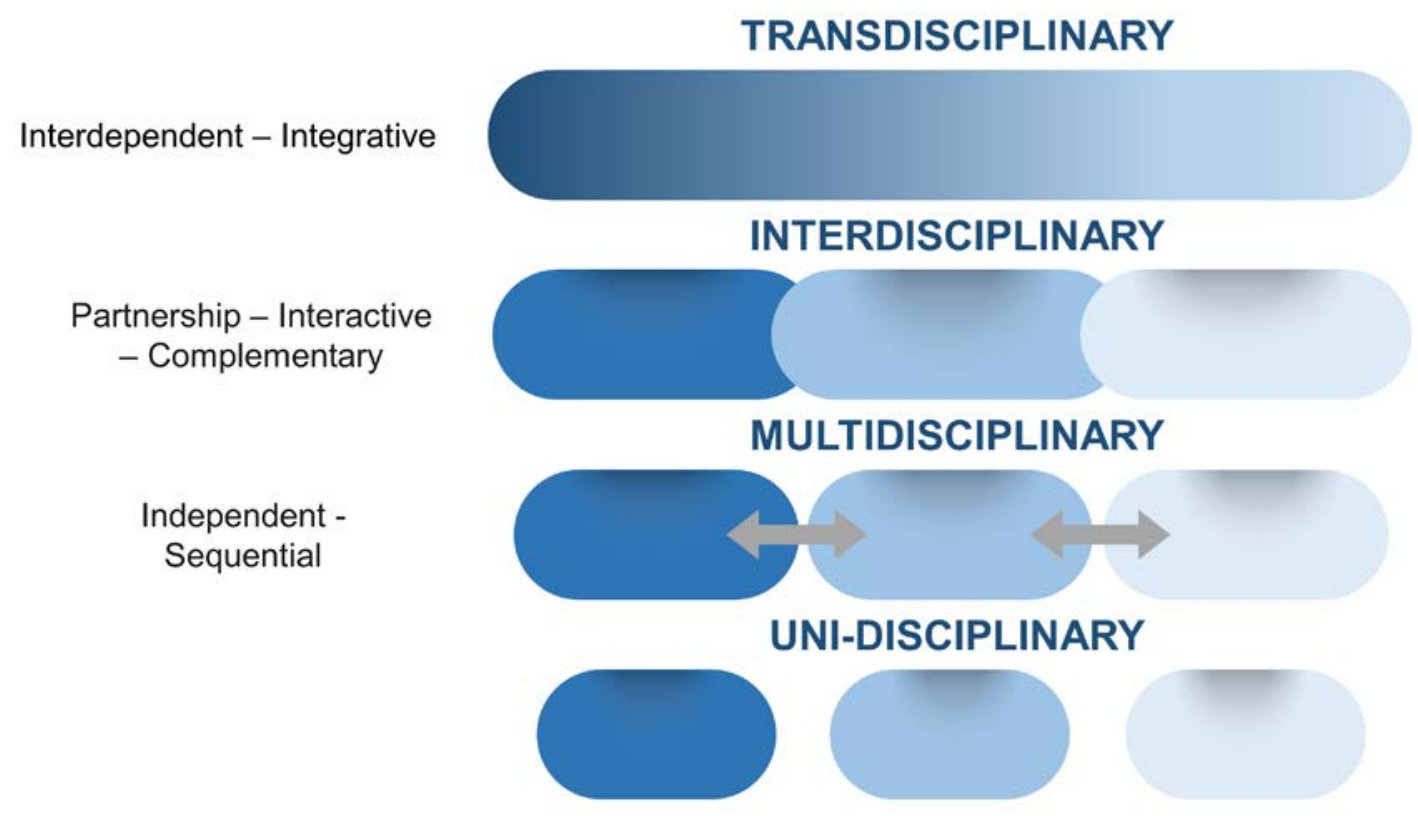

This article is protected by copyright. All rights reserved. 
Figure 2: Interdisciplinary Approach for Repurposing Bisphosphonates for Cancer Therapy.

\section{INTERDISCIPLINARY APPROACH}

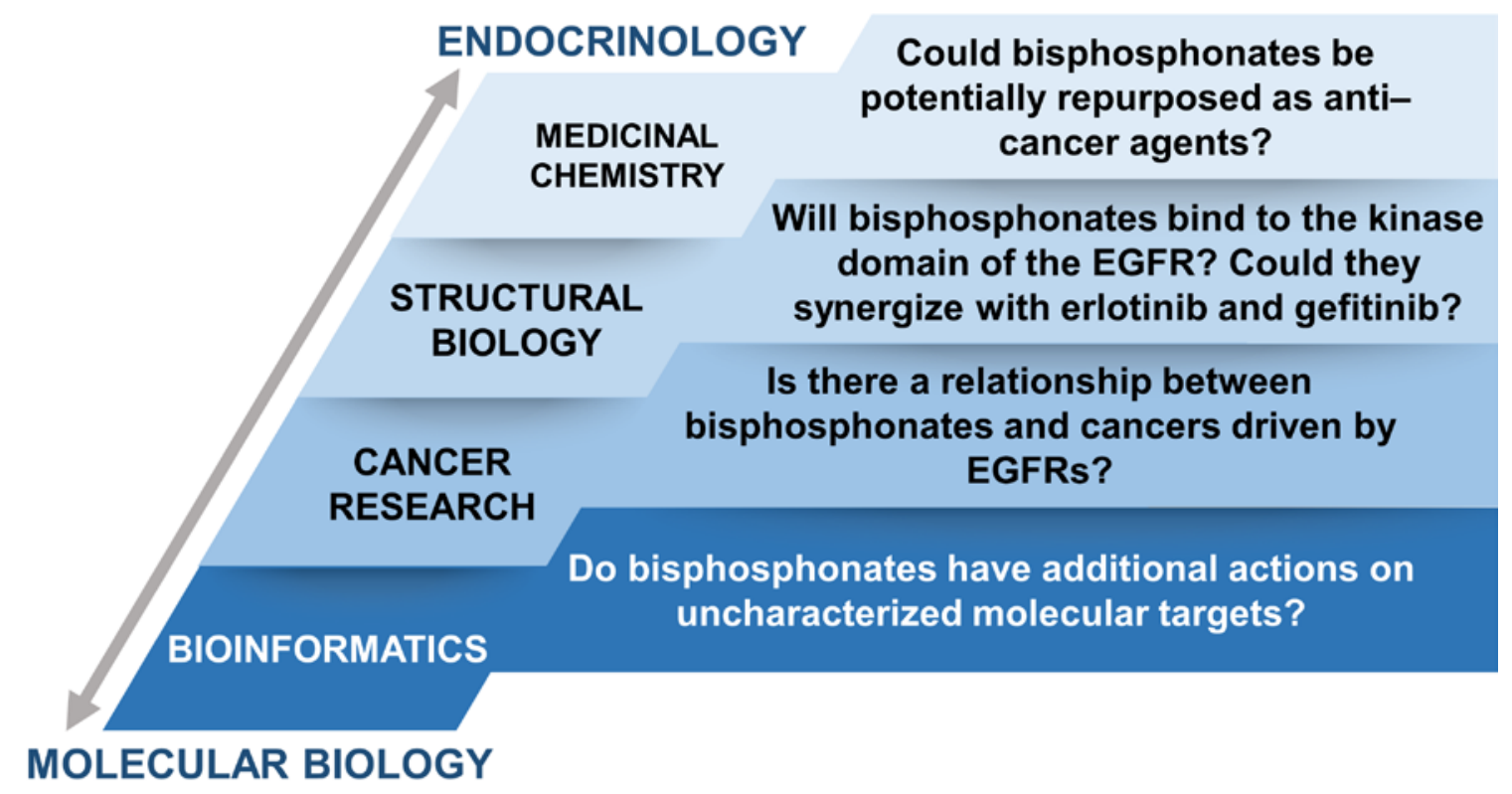

This article is protected by copyright. All rights reserved. 